\title{
Medidas socioeducativas y colaboración interprofesional: entre tensiones y nuevas estructuras profesionales
}

Socio-educational policies and interprofesional collaboration: between tensions and new professional structures

\author{
Catherine Blaya*1 \\ Catherine.blaya@univ-cotedazur.fr \\ Marco Allenbach** \\ marco.allenbach@hepl.ch \\ VALÉRIE ANGELUCCI** \\ valerie.angelucci@hepl.ch \\ FrÉDÉRIQUE REBETEZ** \\ frederique.rebetez@hepl.ch \\ *URMIS, Université Nice Cote d'Azur - LASALE, Haute \\ École Pédagogique du Canton de Vaud, Suiza \\ ** LASALE, Haute École Pédagogique du Canton de Vaud, Suiza
}

\section{Resumen:}

Introducción: este artículo trata de la aparición del trabajo socioeducativo en el ámbito escolar para prevenir el abandono escolar en Suiza (cantón² de Vaud). El término "socioeducativo» se aplica conjuntamente a una serie de situaciones problemáticas recientes, nuevos dispositivos o profesionales adecuados designados para responder a ellas y, también una nueva manera de nombrar algunas de sus actividades. Método: siete Focusgroups que asocian diferentes colaboradores profesionales y luego docentes fueron

\begin{abstract}
:
Introduction: this article aims to investigate the emergence of socio-educational work in the school context in order to prevent early school leaving in Switzerland (Canton of Vaud). The term "socioeducational" is used jointly to refer to problematic situations and resources or professionals devoted to addressing such situations. Finally, the term is also used to refer to certain activities beyond teaching. Method: seven focus groups including specific professionals and teachers were surveyed (i) to conceptualize their repre-
\end{abstract}

1 Dirección para correspondencia (correspondence address):

Catherine Blaya. Directrice: INSPE de I'académie de Nice. 89 bd George V. 06046 Nice Cedex (Francia).

2 Se denomina cantón a cada una de las regiones suizas con gobierno autónomo y capitalidad. La capital del cantón d Vaud es Lausana. (Nota de traducción) 
Medidas socioeducativas y colaboración interprofesional: entre tensiones y nuevas estructuras profesionales

Catherine Blaya, Marco Allenbach, Valérie Angelucci y Frédérique Rebetez

organizados han permitido investigar sus representaciones del trabajo socioeducativo, comprender los desafíos establecidos e identificar algunas condiciones para que los recursos y estrategias movilizados contribuyan al apoyo de los alumnos en dificultad. Cada transcripción integral fue objeto de un análisis temático llevado a cabo por dos investigadores. Resultados: los resultados muestran que el término "socioeducativo" se puede utilizar para designar diversos ámbitos de actuación considerando cada uno de ellos como perteneciente, tanto a las tareas de los docentes, como a otras competencias profesionales. El trabajo socioeducativo aparece como representativo de la relación interprofesional ya que supone un campo de actividad al que contribuye el conjunto de la comunidad educativa.

\section{Palabras clave:}

Socio-educacional; alumnos en dificultad; colaboración; interprofesional. sentations of socio-educational work; (ii) to define perceived challenges; and (iii) to identify conditions which foster the support of disadvantaged children. Findings: the results show that the term "socio-educational" can be used to designate various types of activities which, sometimes, are related to teaching and, some other times, to other professional skills. Socio-educational work seems to represent interprofessional relationships in that it involves the whole educational community.

\section{Key words:}

Socio-educational; schools; disadvantaged children; collaboration; inter-professional.

\section{Résumé:}

Introduction: cet article s'intéresse à l'apparition du travail socio-éducatif dans la sphère scolaire afin de prévenir le décrochage scolaire, en Suisse (canton de Vaud). Le " socioéducatif » désigne conjointement une nouvelle catégorie de situations problématiques, des nouveaux dispositifs ou acteurs professionnels appelés à y répondre, et enfin, une nouvelle appellation pour certaines activités. Méthode : sept focus-groups, réunissant les divers professionnels ressources puis des enseignants, ont permis $d^{\prime}$ 'investiguer leurs représentations du travail socio-éducatif, de comprendre les défis posés par l'articulation entre leurs activités respectives, et d'identifier certaines conditions pour que les ressources mobilisées contribuent à soutenir l'accompagnement des élèves en difficulté. Résultats: les résultats montrent que le terme " socio-éducatif » peut être utilisé pour désigner diverses sphères d'activité chacune étant considérée tantôt comme appartenant au travail enseignant, tantôt comme relevant d'autres compétences professionnelles. Le travail socio-éducatif apparaît comme emblématique de l'intermétiers, car représentant un champ d'activité auquel contribue l'ensemble de la communauté éducative.

\section{Mots clés:}

Socio-éducatif; écoles; élèves en difficulté; collaboration; interprofessionel.

Fecha de recepción: 04-02-2020

Fecha de aceptación: 05-03-2020 


\section{Introducción}

Los profesionales de la escuela se encuentran enfrentados a múltiples presiones y la introducción de medidas, cuyo objetivo sea la gestión de "lo social" en el ámbito de la escuela para prevenir el abandono escolar implica un análisis de la representación mental de la profesión docente, a veces, reducida a la mera transmisión de conocimientos (Maroy, 2018). Conlleva también la colaboración entre diferentes profesiones. La escuela acoge a alumnos de todos los medios sociales $y$, tanto los poderes públicos como las familias e incluso los jóvenes mismos, hacen hincapié en las dificultades que tiene para ofrecer oportunidades iguales de éxito.

La investigación se inscribe en un proceso de seguimiento de un centro escolar en el cantón de Vaud, que desea documentar la puesta en marcha de un dispositivo de apoyo socioeducativo; pero teniendo en cuenta también el conjunto de ayudas que se ofrecen desde una perspectiva de inclusión. A partir del discurso del grupo de profesionales ${ }^{3}$, nos interesamos por la representación mental del trabajo socioeducativo, los recursos que el centro escolar ofrece y su coordinación, sin olvidar las dinámicas que intervienen en las relaciones interprofesionales. Un cierto número de obstáculos, tanto a nivel de imágenes mentales o identidades profesionales como en la práctica de su colaboración, interpela a los profesionales y esto les lleva a redefinir su papel y funciones en el marco de sus interacciones. Manifiestan así, no solamente las dificultades que encuentran, sino también los "bricolajes" (en el sentido de LéviStrauss, 1990), su capacidad de improvisación, es decir, las prácticas de colaboración que inventan y negocian entre los profesionales diversos que intervienen. Nuestra iniciativa no parte de una definición a priori del trabajo socioeducativo, atribuido a una categoría profesional. Se interesa por las representaciones mentales del trabajo socioeducativo y por la manera en que se relacionan entre sí, las actividades del conjunto de profesionales de la escuela.

Los objetivos son:

1/ Entender mejor las representaciones de los actores en la comunidad escolar con respecto al trabajo socio-educativo.

2/ Comprender cómo se articulan y apoyan las actividades socioeducativas a los diferentes profesionales de la escuela.

3 Docentes oficiales y personal de apoyo (profesores especializados, personal sanitario y social, etc...) 
3/ Comprender cuáles son los obstáculos y facilitadores de la implementación de actividades socioeducativas.

\section{"Inserción de lo social" en el ámbito escolar y nueva configuración de la colaboración interprofesional}

Sin entrar en detalles sobre las reformas pasadas y actuales que están transformando el paisaje de la educación y la enseñanza en Suiza y en el mundo, observamos una tendencia de las políticas públicas cuyo objetivo es promover la educación y el éxito para todas y todos (UNESCO, 2016). A instancias de la competitividad de los sistemas educativos y los centros escolares, y también de las políticas de reducción de la tasa de abandono escolar en los países de la OCDE, se observa que los conceptos de integración y de inclusión, en vigor en las políticas escolares, se han ampliado para incluir las nociones de eficacia y éxito escolar para todos. La escuela actual se encuentra inmersa en las tensiones que ocasionan las recomendaciones de la escuela inclusiva,por una parte, $y$, los imperativos de la escuela que pretende obtener excelentes resultados por otra, con el impulso de la nueva administración pública (Marcy, 2018).

Durante las últimas décadas, las políticas de integración y de inclusión escolar, tanto en Suiza como en el resto del mundo, han contribuido al desarrollo de propuestas, cuyo objetivo es hacer frente a las necesidades específicas de los alumnos en términos de dificultad de aprendizaje, integración escolar y discapacidades. Medidas de apoyo en el campo "socioeducativo" fueron introducidas en cantón de Vaud que se destinan a un alumnado de alto riesgo de abandono escolar. Estas nuevas formas de apoyo "introducen lo social" en el ámbito escolar. Lo que nos lleva a considerar lo social, según Maroy (2018) no solo bajo el prisma de la precariedad social y económica, sino también a través del análisis de situaciones que se consideran "fuera de la norma", y que ponen de manifiesto la "expectativa sobre las normas" en vigor en el sistema educativo,en el origen de una nueva composición de modalidades de trabajo y colaboración en el ámbito escolar.

En efecto, estos cambios políticos implican una transformación importante del contexto escolar. Exige un trabajo de concertación y la construcción de alianzas educativas inter e intra-institucionales. Esta 
evolución, patente en la mayor parte de los sistemas educativos occidentales, conlleva, poco a poco, la modificación de la figura tradicional del docente como único profesional a bordo "(...) para dejar su lugar a la figura de un profesional que ejerce en un centro escolar y que se confronta al aumento de una serie de colaboradores específicos" (Marcel, Dupriez, \& Périsset Bagnoud, 2007, p. 8-9). Los centros escolares están sometidos a un doble mandato en el sentido de que, al mismo tiempo, deben ofrecer una igualdad de acceso, de tratamiento, de promoción de la equidad mediante la aplicación de medidas de discriminación positiva y responder a las exigencias de eficacia y a las directrices impuestas por las instancias supranacionales que refuerzan la competitividad para garantizar el rendimiento de los sistemas educativos (Maroy \& Voisin, 2013). Esto genera un movimiento doble y paradójico que incluye, por una parte, una división creciente del trabajo, que va en paralelo con el mandato de colaboración y, por otra, la multiplicación de los cometidos que se esperan de los docentes, en particular en lo que concierne a las tareas socioeducativas (división interna de su trabajo) y paralelamente a la multiplicación de agentes no-docentes.

A los docentes se les considera, al mismo tiempo, como "colega" para el personal de apoyo que interviene en la escuela y como "beneficiarios" de su trabajo, puesto que se tiene en cuenta el apoyo que reciben para ayudar a los estudiantes. En consecuencia, se nos plantea la cuestión de este apoyo tal como lo perciben los docentes (Gabola \& Curchod-Ruedi, 2017) y de las formas de trabajo compartido o más concretamente de los espacios interprofesionales (Thomazet \& Merini, 2014), así como la cuestión de la resistencia al trabajo en equipo interprofesional. El desarrollo de tales colaboraciones interprofesionales implica un cambio de visión de la propia cultura profesional, compartir informaciones sobre el alumno y su seguimiento, construir relaciones de confianza mutua respetando los códigos deontológicos respectivos y elaborar proyectos de trabajo coordinado (Friend, Cook, Huerley-Chamberlain, \& Shamberger, 2010). La coexistencia de diferentes disciplinas profesionales puede dificultar el hecho de compartir, no solo el trabajo, sino también la responsabilidad y los valores que la fundamentan (Henneman, Lee \& Cohen, 1995). Sin embargo, la colaboración puede favorecer la contribución de cada uno en la coordinación educativa del seguimiento de los alumnos (Allenbach, 2019).

Las cuestiones de la investigación, recogidas en este artículo, son: 1/ 
¿Cuáles son las representaciones mentales de los actores que integran la comunidad escolar en relación al trabajo socioeducativo? 2/ ¿Cómo se articulan (coordinan) las actividades socioeducativas entre los diferentes profesionales de la escuela? 3/ ¿Cuáles son las condiciones en las que los recursos movilizados contribuyen a apoyar el trabajo de los profesionales implicados?

\section{Contexto de la investigación}

Recientemente, el departamento de instrucción pública del cantón de Vaudha establecido unos dispositivos de intervención socioeducativa. El centro escolar en el que se ha desarrollado la investigación continúa, desde hace varios años, con proyectos para desarrollar su capacidad inclusiva. Los recursos pedagógicos están organizados para favorecer la implementación de la co-enseñanza entre docentes ordinarios y docentes de apoyo. Además, han creado otro dispositivo de "medida socioeducativa" en el que intervienen otros docentes y un educador. Su finalidad es apoyar al conjunto escolar en situaciones de crisis, así como elaborar proyectos a medio plazo relacionados con problemáticas relativas al ambiente de clase y a las dificultades comportamentales. También existen otros recursos distribuidos por el cantón: docentes de cursos intensivos de francés para los alumnos alófonos; psicólogos, logopedas (ortofonistas) y especialistas en psicomotricidad en el medio escolar; enfermeros y mediadores escolares; docentes especializados itinerantes; asistentes de integración. Por último, los consejeros entre escuela y familia (asistentes sociales o educadores especializados) financiados por el Ayuntamiento.

\section{Elaboración de las líneas de investigación con el centro escolar}

Se efectuaron cuatro reuniones entre los investigadores y el consejo de dirección del centro escolar, compuesto por un director y cuatro "doyennes-s"(Término que en el cantón de Vaud designa a los docentes que desempeñan tareas de dirección en apoyo al director/la directora). A pesar de la impresión satisfactoria de los docentes respecto a este servicio, el consejo de dirección manifestó su preocupación en relación a la gran cantidad de intervenciones solicitadas al mismo y al agotamiento expre- 
sado por algunos de los integrantes de dicho servicio. Nuestros interlocutores manifestaron el deseo de una visión externa que contribuyera a una mayor comprensión del modo de funcionamiento, a partir de la representación mental de los profesionales.

Acordamos adoptar como método de trabajo el focus group (Kitzinger, Markova \& Kalampalikis, 2004) que permite la participación de todos los actores que deseen implicarse en la investigación y ofrecer una ocasión de diálogo sobre esta temática, contribuyendo al análisis de las problemáticas abordadas y al desarrollo funcional del centro escolar.

Consideramos la situación particular del personal de apoyo, acción que nos llevó forzosamente a una especie de evaluación de su actividad, cuestionándonos el modo en el que su intervención favorece el desarroIlo del sentimiento decapacidad en los docentes. En primer lugar, organizamos reuniones con el personal de apoyo para poder asociarlo también a esta investigación, compilando sus preocupaciones y preguntándoles cuáles eran las cuestiones que deseaban que formulásemos a los docentes. Aparte de esta distinción entre personal de apoyo y docentes, fomentamos una participación mixta en los focus groups para favorecer los intercambios entre los docentes que trabajan en diferentes ciclos, y entre el personal de apoyo de diversos ámbitos profesionales. Un formulario de entrevistas permitió estructurar el discurso de los participantes en torno a los temas que formaban parte del objetivo de la investigación (Van Campenhoudt \& Quivy, 2011): 1/ ¿En qué medida su actividad contribuye a un trabajo socioeducativo?; 2/ ¿Qué logros encuentra usted en la implementación de los apoyos socioeducativos?; 3/ ¿Qué dificultades encuentra en la implementación de los apoyos socioeducativos?; 4/ ¿Qué supone este dispositivo de medidas socioeducativas para usted?; 5/ ¿Cómo se ha sentido en esta sesión?

En segundo lugar, realizamos sesiones con los docentes. Tuvimos especial cuidado en garantizar el anonimato de los participantes. Dirigimos siete focus groups: tres con el personal de apoyo (17 miembros) y cuatro con los docentes (19 miembros). Los diecinueve docentes representaban todos los niveles de enseñanza (de primero a sexto de enseñanza obligatoria), en una proporción ligeramente mayor de docentes de primer ciclo (primero a cuarto de primaria). Los diecisiete miembros del personal de apoyo representaban cada una de las funciones, profesiones y pertenencia institucional, a razón de uno a tres representantes de cada 
función, a excepción de los logopedas. El número de hombres, dos, no se aleja del porcentaje de personal masculino que trabaja en este centro escolar.

\section{Deontología}

Este estudio se basa en los principios promulgados en el código ético de la investigación para las escuelas superiores de pedagogía (CDHEP, 2002). La iniciativa se presentó en una reunión plenaria organizada por el centro. Se informó a los participantes sobre los objetivos de la investigación y la utilización posterior de los datos obtenidos. La participación fue voluntaria y garantizamos el anonimato de todos, tanto fuera como en el propio centro. Al comienzo de cada focus group pedíamos el consentimiento de los participantes para ser grabados, precisando el uso y las condiciones de utilización de los ficheros auditivos. No encontramos oposición alguna a esta manera de proceder. Para preservar el anonimato de los participantes, los extractos utilizados en la presentación de resultados se catalogaron así: FGR + número del focus group para el personal de apoyo y FGE + número del focus group para los miembros del grupo de docentes.

\section{Análisis de los datos obtenidos}

Los siete focus groups se grabaron y transcribieron de manera literal e integral. Fueron objeto de un análisis temático. Los tres investigadores implicados en el proceso hicieron una primera recopilación de datos con vistas a armonizar el enfoque del análisis. A continuación, cada focus group fue objeto de otra recopilación y de un análisis temático llevado a cabo por dos investigadores. Nuestro enfoque de análisis se basó en "una dialéctica de los movimientos inductivo y deductivo"susceptible de concretar las representaciones mentales de los actores, que no pueden "ser consideradas ipso facto como un conocimiento científico, ni incluso un conocimiento válido" (Crahay, Wanlin, Issaieva, \& Laduron, 2010, p. 47). 


\section{Resultados y Discusión}

\section{Representaciones mentales del trabajo socioeducativo en la profesión docente}

La representación mental del trabajo socioeducativo que tiene el personal interrogado es vasta y procede de varios campos:

- De la vida de clase: se describen las iniciativas que permiten trabajar sobre el ambiente de la clase, el "vivir juntos", las reglas de vida, pero también la aceptación de las diferencias, la comprensión de las emociones. Se evocan las intervenciones como la mediación en los conflictos.

- De las relaciones con la familia: se evocan, por una parte, los esfuerzos para crear un vínculo con los padres y, por otra, las dificultades, a veces, para ponerse de acuerdo, por ejemplo, en la aceptación de nuevos apoyos.

- Acciones y preocupaciones centradas en un alumno, ya sea para saber "gestionar" sus momentos de crisis o comportamientos difíciles recurrentes.

Los docentes pueden designar, como emanando de lo "socioeducativo", actividades y preocupaciones profesionales de toda índole. Se constata, por una parte, un cierto malestar -atribuido a menudo a las políticas de inclusión impulsadas por los acuerdos de Salamanca (1994)en relación al seguimiento de alumnos considerados como "difíciles" o "en dificultad". La inclusión escolar, percibida desde un punto de vista filosófico como una iniciativa honorable, suscita un sentimiento de sobrecarga de trabajo. Las respuestas muestran que aun si los docentes se refieren a una definición de la actividad docente centrada en la transmisión de conocimientos, se implican también en los apoyos calificados de socioeducativos.

"No digo que ya no enseñemos, (...) pero pienso que el espacio socioeducativo alcanza cada vez más importancia a lo largo de los años. Cada vez, tenemos que implicarnos más y ejercer otro papel. Lo socioeducativo es nuestra misión también, pero deberíamos... es sobre todo enseñar, la base" (FGE2). 
Medidas socioeducativas y colaboración interprofesional: entre tensiones y nuevas estructuras profesionales

Catherine Blaya, Marco Allenbach, Valérie Angelucci y Frédérique Rebetez

El trabajo socioeducativo aparece ya como parte integrante de la profesión docente, al mismo tiempo inevitable y periférica, que la aleja de su verdadero oficio y de su campo de competencias.

\begin{abstract}
"Así pues, yo veo también el lado socioeducativo en el hecho de vivir juntos, y pues bueno... pienso que lo hacemos, lo hacemos cotidianamente en nuestra práctica, a través de diálogos u otras cosas (...) las diferencias que... creadas por las familias, pienso que, para nosotros, es a veces difícil intervenir cuando es verdaderamente grave, porque afecta a la educación que los padres han dado y... creo que eso sale de nuestra ámbito, del ámbito de la clase. Ahí es donde se debe intervenir con los asistentes sociales u otros." (...) (FGE4).
\end{abstract}

De este modo, el trabajo socioeducativo puede designar la gestión necesaria para la cohabitación en clase, siendo esto plenamente responsabilidad de la escuela, o, al contrario, la corrección de una educación familiar percibida como alejada de las normas escolares o incluso defectuosa:

"Tengo alumnos que pasan seis horas al día delante de las pantallas. En fin, verdaderamente, me ocupo de todo: comer, dormir. Yo me implico, esto quiere decir que no voy a... juzgar, pero vamos a dialogar al respecto (...). Para los alumnos de primero (...) todo se definió a la entrada escolar y, de pronto, es como una... estructura ya establecida. Y luego, eso no impide que, a veces, incluso con todo eso, haya necesidad de refuerzo (...) y es, de hecho, un trabajo a largo plazo" (FCE4).

Este trabajo, asociado a la brecha existente entre las representaciones mentales que se tienen del papel educativo de los padres, puede percibirse como una fuente de cansancio e incluso de agotamiento.

Globalmente, el trabajo socioeducativo forma parte de las representaciones mentales del papel del docente. No obstante, el sentimiento de desprestigio de la identidad profesional y la pérdida de la noción básica de la profesión aparece a menudo en los discursos y refleja estrés y falta de poder de acción (Payet, Deshayes, Rufin, Pelhate, 2018).

Esto tiene una influencia negativa sobre las capacidades de los docentes para aportar un apoyo socioeducativo a los alumnos (Lynn, Mc Kay \& Atkins, 2003).También puede conllevar efectos doblemente perjudiciales ya que el alumno que tiene necesidad de apoyo no puede obtenerlo, 
lo que refuerza la dificultad tanto para el alumno como para el docente. Una de las docentes confiesa esta dificultad de la que responsabiliza a algunos alumnos en particular. Estos alumnos aparecen como fuente de perturbación que la obligan a ejecutar actividades alejadas de su idea de la enseñanza:

"Pienso que, cuando se habla de grupo de clase, puede ser que incumba al trabajo del docente,el hecho establecer bases, reglas. Pero pienso que es en relación a ciertos alumnos específicos que tienen concretamente grandes dificultades, que necesitan un seguimiento y que, en la mayoría de los casos, ya lo tienen pero que nos obligan o que crean situaciones en las que nos sentimos obligados... de parar y de hablar, ya sea con el alumno en cuestión, ya sea con toda la clase. Digamos que el alumno que tiene muchas dificultades es quien nos obliga de pronto, a buscar soluciones a nivel de pura educación y a pasar tiempo con los padres (...) a intentar encontrar a los profesionales que podrían ayudarnos porque nos decimos que esto ya no forma parte de nuestro oficio." (FGE2)

El análisis de las informaciones recopiladas en los focus groups permite demostrar que los docentes se sienten implicados en las cuestiones de orden sicológico y social. Se responsabilizan de una parte socioeducativa de su oficio, pero esta se percibe como una actividad fuera de sus funciones y una sobrecarga. Por eso, las fronteras oscilantes del trabajo socioeducativo necesitan ser definidas, caso por caso, en las situaciones concretas. Una parte de los comentarios nos lleva a reflexionar a propósito de los efectos de la llegada del apoyo "socioeducativo" a la escuela como un nuevo servicio que refuerza los recursos ya existentes, $y$, por supuesto, una nueva profesión.

\section{El trabajo socioeducativo considerado como un apoyo específico}

En los focus groups en que intervenían los profesionales de recursos de apoyo, cada vez que un participante presentaba una actividad socioeducativa como específica de su función, otros participantes explicaban que ellos también se ocupaban de ese tipo de apoyo. Todo esto confirma que el trabajo socioeducativo representa un espacio multidisciplinar más que un trabajo específico reservado a una única función o profesión.

La llegada de educadores a los centros plantea, sobre todo, una serie 
Medidas socioeducativas y colaboración interprofesional: entre tensiones y nuevas estructuras profesionales

Catherine Blaya, Marco Allenbach, Valérie Angelucci y Frédérique Rebetez

de cuestiones, en relación con el papel y la función que desempeñan. Así lo destaca la dirección del centro en una de las reuniones de preparación para los focus groups:

"El nuevo colaborador contratado responde a un proyecto de educador, pero no de docente, lo que puede plantear una dificultad sobre todo en lo que respecta a los niños con dificultades de orden cognitivo. Y además ¿cómo "injertar", añadir a un colega que llega de un mundo completamente diferente?" (FGE3)

Pero independientemente de su función, la demanda de un colaborador de apoyo supone,al mismo tiempo, un recurso a disposicióny un estrés.

"Es un arma de doble filo. Al comienzo, es un apoyo y una presión. Pienso que tener a alguien en clase es, cuanto menos, un estrés suplementario (...) Lo socioeducativo significa, a pesar de todo, educadores, o sea, alguien que tiene una formación, es ya un punto positivo. Ahora bien, en relación a las ayudas, a veces es una carga suplementaria... lo que encuentro positivo, bien, es para el niño. Este año, cuando el educador está presente, es magnífico, se pueden gestionar todas las crisis." (FGE1)

El colaborador socioeducativo aparece, a veces, como un participante momentáneo al que se le puede solicitar apoyo para que se encargue de un alumno cuyo comportamiento perturba al resto de la clase. Pero insisten también en el hecho de que construir una intervención que adquiera significado más allá de la simple "reparación momentánea" supone energía, tiempo, trabajo de coordinación y de elaboración de acuerdos. Además de la energía necesaria para organizar las intervenciones, la presencia de estos nuevos colegas en la clase, no facilita automáticamente, una colaboración efectiva:

"Y bien, mi clase se beneficia de una ayuda socioeducativa, pero el día en que yo no trabajo, no tengo contacto directo con esa persona." (FGE3)

El campo de acción de los colaboradores socioeducativos en la escuela se sitúa completamente en la interdisciplinaridad puesto que ningún acto profesional les está especificado, contrariamente a los psicólo- 
gos, enfermeros o médicos, únicos habilitados a efectuar ciertos gestos profesionales como, por ejemplo, control médico, un informe, etc. En cambio, quizá sea la profesión de educador especializado la mejor equipada en términos de conocimientos y gestos profesionales para elaborar acuerdos multidisciplinares y proyectos que impliquen al conjunto de todos los actores.

Pero los docentes lamentan muy claramente que un educador no conozca el mundo escolar, los aspectos pedagógicos de la profesión docente, incluso los planes de estudio, como lo confirma el extracto de la siguiente entrevista:

"Tiene toda la dimensión educativa, pero no el lado docente. Puede ser una dificultad ya que algunos niños presentan dificultades cognitivas." (FGE4)

Nos encontramos ante una situación multidisciplinar en torno al mismo alumnado, pero en la que cada cual trabaja en paralelo y de manera autónoma, a partir de una atribución de alumnos en función de sus "necesidades específicas".

La ambigüedad en cuanto al papel de los educadores se destaca ya en un informe realizado por Soussi, Nidegger y Schwob (2014) para el cantón de Ginebra, según el cual, "Ios educadores ginebrinos parecen ser una excepción ya que ejercen una función socioeducativa principalmente, aun pudiéndose constatar una cierta ambigüedad en la definición de sus funciones, que los sitúan, a veces, en el límite del ámbito pedagógico ("contribuye por sus acciones al desarrollo [...] de los aprendizajes" o "permite una acción preventiva [...] del fracaso escolar")."

La principal preocupación reside en la coordinación entre los docentes y el conjunto de medidas de apoyo propuestas. Estas tareas, que implican nuevas colaboraciones, la puesta en marcha de modos de comunicación y trabajo diferentes, así como el desarrollo una cultura interprofesional, necesitan mucho tiempo de dedicación. Por eso algunos profesionales las perciben como causa de estrés suplementario y no de apoyo.

\section{Los obstáculos de la colaboración}

Tanto los docentes como el personal de apoyo socioeducativo aprecian un gran número de las colaboraciones. No obstante, el intercambio de visiones múltiples se percibe también en ocasiones como "estéril y com- 
plicado". Los docentes tienen la impresión, a veces, de que las dificultades encontradas en clase no las comprenden los colaboradores de apoyo. Por parte del personal de apoyo, se destaca la falta de comunicación y el hermetismo entre los diversos profesionales. Partiendo de la necesidad de ser prudentes en términos de secreto profesional y confidencialidad, los colaboradores entrevistados estiman que una comunicación mejor favorecería el proceso del apoyo.

"Hay (...) cosas que se pueden decir y otras que no. Es la protección del ámbito privado. Pienso que sería bastante lógico que yo sepa que, de entre los alumnos a los que les hago seguimiento, hay algunos que ya están en manos (...) de la asistente social, la especialista de sicomotricidad o el psicólogo. Para mí, sería un ahorro de tiempo y podríamos compartir nuestras visiones de las cosas, elementos que pueden ser importantes para la gestión de apoyo (...)." (FGR1)

Esta falta de comunicación se explica también por la urgencia de las situaciones y por el número de alumnos que están en seguimiento. Por eso, una integrante del equipo de recursos subraya que, con alrededor de quinientos alumnos que seguir, y contratada a tiempo parcial (50\%), se ocupa de las situaciones sin tener tiempo de contactar con los colegas. Esto confirma la representación mental del trabajo de apoyo de alumnos de modo unilateral. Mientras que, desde una perspectiva que reconoce los espacios comunes interprofesionales, el hecho de informarse de si el joven se beneficia ya de un recurso de apoyo podría contribuir, en gran medida, a una economía de medios y de energía:

"Te hayas ante un dilema entre las tareas que hacer, el grado de implicación que tienes que ofrecer a tal o tal alumno, ¿vale la pena? Y, de pronto, te planteas, pero, de hecho, ya está en seguimiento por otro lado, quizá no valga la pena que..." (FGR1)

El modelo médico-individual, que atribuye un especialista a cada necesidad particular, desemboca en una multiplicación de personas que intervienen y hace imposible la colaboración:

"Yo puedo comprenderlo, es bastante paradójico porque es: "necesito ayuda, ¡vengan a ayudarme!", pero al mismo tiempo (...) estos niños necesitan un 
apoyo especializado, entonces, eso exige la intervención de otros especialistas $y$ entonces, es verdad que, al final, esto es demasiado. Cuando trabajamos a veces en red, somos doce alrededores de una mesa." (FGR1)

Las ayudas que recibe el docente pueden representar una dificultad suplementaria, sobre todo, si no existe un buen entendimiento con el o la colega. De este modo, el colega no docente puede percibirse como un ignorante de la relación pedagógica, del mundo escolar o de la realidad de la clase. Aquí, el trabajo de coordinación, de configuración de nuevas relaciones, de nuevos gestos y el hecho de compartir el aula se perciben como forzosos, obligatorios, incluso como una pérdida de tiempo. En un enfoque racional entre implicación y ventajas obtenidas a corto plazo, tal como se pueden percibir, algunos docentes optan por una gestión de las dificultades en solitario.

La colaboración interprofesional reside en intercambios voluntarios de análisis, de conocimientos y de competencias que favorecen así la apertura a diversos espacios disciplinares. Pero todavía, algunos perciben la clase como un territorio privado. Esto se debe, en parte, al cambio de situación profesional que está lejos de las prácticas habituales o de las representaciones mentales de la profesión docente (Lantheaume, 2007). En efecto, para colaborar es necesario una introspección sobre las propias maneras de trabajar, adquirir nuevas competencias y cambiar de posición, lo que implica apoyo y coordinación por parte de los equipos de dirección.

\section{Sobre la importancia de la dirección y la coordinación}

Los focus groups revelan, a pesar del reconocimiento de una fuerte implicación por parte del equipo de dirección, una sensación confusa, en relación a la coordinación de las acciones de apoyo socioeducativo y los papeles respectivos. Hecho que puede generar un sentimiento de postergación:

"Los miembros de la dirección tampoco lo tienen claro en el sentido en que todo es vago, confuso en relación al papel de cada uno." (FGR2)

Las tentativas de objetivar y coordinar intervenciones, a través de análisis de situaciones recogidas en formularios que deben completar, 
Medidas socioeducativas y colaboración interprofesional: entre tensiones y nuevas estructuras profesionales

Catherine Blaya, Marco Allenbach, Valérie Angelucci y Frédérique Rebetez

se perciben como una sobrecarga administrativa e incluso una falta de confianza por parte de la jerarquía:

"Pero las dificultades, según mi punto de vista, son verdaderamente de coordinación.

Sí, es también que yo no gozo de la confianza de mi doyenne en relación a mis dificultades." (FGR2)

El trabajo de concertación y coordinación, de co-construcción y de seguimiento de los proyectos, poco conocido o poco reconocido, resulta a menudo vano, cuando falta un protocolo de acción:

"Con el SPJ4, con todo el mundo, nunca se aclara quién se encarga del informe. Nunca hay nada en claro: es la sicóloga, es el docente, o es... nada,se dice que se hace, pero...". (FGR2)

Además, las dificultades de coordinación y la falta de concertación pueden suscitar una impresión de patchwork y de explosión de tareas y, por otra parte, resaltar, por comparación, el funcionamiento de las instituciones especializadas:

"Yo debo confesar que, personalmente, -sin embargo, yo trabajo para la integración- encuentro que es muy delicado porque es como si se hicieran trocitos de ayuda: a cualquier sitio que mires encuentras un poquito de apoyo, periodos cortos. Y entonces, de hecho, lo que yo ofrecía en algunas buenas instituciones entre comillas (...) era un equipo que trabajaba en forma de supervisión, eran concretamente encuentros semanales, algo que la escuela no puede ofrecer." (FGR1)

Clarificar las dificultades e invitar al diálogo sobre los problemas encontrados sin temor a ser etiquetado como mal docente son clave para la colaboración. Parece difícil poder elaborar una cultura común basada en un sentimiento de falta de confianza, de cosas no dichas y también de vergüenza de ser juzgado. Todo esto no se improvisa y los centros escolares tienen, en este aspecto, una responsabilidad para asegurar una satisfacción mayor de las personas implicadas (Mahieu, 1992). El esfuerzo vale la pena porque, cuando los equipos de dirección apoyan la

4 Servicio de protección de la juventud 
colaboración, se constata un aumento de la capacidad de implicación en las prácticas innovadoras y la participación del conjunto de todos los actores en el cambio (Bryk, 2015).

\section{Logros y resultados satisfactorios: una profesión en evolución}

La colaboración en clase se percibe como propicia para establecer relaciones de confianza y cambiar los puntos de vista que se tienen de las dificultades, al tiempo que permite compartir el estrés y estar más atentos. El sentimiento de eficacia está motivado también por la impresión de colaborar en la mejora de la situación de los niños en dificultad y poder tomar distancias de las situaciones experimentadas para captarlas mejor:

"(Los) consejos de clase y todo eso, bueno, hace avanzar las cosas en el interior. En el interior de la clase, todo lo que se pone en marcha hace avanzar las problemáticas, y además da un nuevo enfoque a las problemáticas (...) permite tener ese momento de... también de seleccionar "¿de qué hablamos en este momento?" "¿De qué te sientes capaz de asumir solo el resto de la semana?"

Los profesionales del equipo de recursos se sienten satisfechos de poder contribuir a la mejora de las condiciones de trabajo y de ambiente de clase, tanto de los alumnos como de los profesores, lo que refuerza el sentimiento de eficacia y confirma la existencia de verdaderas formas de trabajo compartido (Tardif \& Borges, 2009).

"Se ha construido todo un programa con la docente (...) me gusta ir en busca de las necesidades, las peticiones de los docentes antes de establecer una intervención, puesto que ella, ella sabía exactamente lo que no funcionaba, dónde se volvía complicado para ella, y entonces elaboramos todo un programa sobre eso y es verdad que esto ha cambiado completamente todo (...). El ambiente de la clase ha cambiado mucho y eso se situaba, verdaderamente,en el ámbito socioeducativo." (FGR3)

Finalmente, la colaboración de las personas de apoyo en la clase se puede percibir como una ayuda nada despreciable. Se reconocen sus conocimientos como una plusvalía y sus intervenciones, como una fuente de alivio para los docentes, de reafirmación de sus propias 
competencias y de distanciamiento de las situaciones experimentadas.

"Bueno, yo tengo personas de apoyo en mi clase. Estoy de acuerdo contigo, son personas competentes, en fin, en todo caso este año (...). Estoy muy satisfecho. Me ha ayudado mucho, nada más que el hecho que ella estuviera presente, ya me sentía menos solo. Y también me reconfortó en el sentido de que lo que yo hacía no era erróneo... no tenía que hacerlo de otra manera (...) también me ha permitido tomar distancia un poco, digamos cargarme un poco de energía (...) Me ayuda mucho personalmente." (FGE4)

La configuración de intervenciones elaboradas en colaboración permite a los docentes sentirse beneficiarios de las ayudas aportadas por los colegas de apoyo y no como una carga que soportar. Las medidas de apoyo socioeducativas propuestas en el centro se perciben, no solo como una ayuda para el alumno,sino también para los docentes, como un aporte innovador y de motivación. Estos testimonios muestran que la colaboración interprofesional puede contribuir a un sentimiento de mejora y de desarrollo profesional tanto en los docentes como en los diferentes colaboradores de la escuela. No obstante, las experiencias presentadas como positivas, se acompañan a menudo de la descripción de un trabajo de coordinación que permite reflexionar juntos sobre la evaluación de las necesidades, las modalidades de intervención, las prácticas aplicadas por los diferentes actores y la evolución de los proyectos. La expresión de los resultados satisfactorios son terreno abonado para un cambio de prácticas y de innovación que constituyan, a pesar de las dificultades encontradas, las bases de una mejora de las condiciones de aprendizaje y de trabajo para el conjunto de la comunidad educativa.

\section{Conclusión}

El objetivo de esta investigación era comprender mejor como los profesionales se describen en relación a los apoyos socioeducativos, así como sus experiencias y el sentido que les han dado a la colaboración interprofesional en un contexto de refuerzo del apoyo socioeducativo en el ámbito escolar. Se han identificado diversas dificultades como los imperativos de tiempo, la fragmentación de la colaboración, las dificultades de coordinación y el sentimiento de sobrecarga de trabajo. 
También se han catalogado los resultados satisfactorios: sentimiento de utilidad y eficacia en la comprensión de situaciones problemáticas y el seguimiento de alumnos con necesidades específicas. La aparición de lo socioeducativo en la escuela puede reforzar la división del trabajo, o, por el contrario, poder salir de ese círculo vicioso de dos maneras: 1/ reconociendo lo socioeducativo como un espacio interprofesional que implica al conjunto de los profesionales; 2/ aportando una nueva cultura profesional dirigida por los trabajadores sociales cuya gestión de las dificultades y conocimientos se basan en el trabajo de concertación.

La asociación de la intervención de diferentes profesionales en situaciones complejas individuales (alumnos) o colectivas (clase) permite la creación de alianzas educativas con el fin de abordar las situaciones más allá de las competencias individuales o específicas de una profesión, en un enfoque más globalizado y, en consecuencia, más completo de lo que el problema plantea (Blaya, Gilles, Plunus \& Tièche Christinat, 2011). Uno de los desafíos de la educación del siglo XXI consiste en pasar de una colaboración superficial a una iniciativa que permita a los docentes, trabajadores sociales o personal de salud desarrollar juntos estrategias de intervención que faciliten el desarrollo de la inclusión de todos los alumnos, apoyando la identidad profesional, la autoestima y el sentimiento de eficacia de cada uno.

Los informes recopilados revelan la existencia de espacios comunes entre las diferentes funciones que componen los servicios socioeducativos. La colaboración interprofesional necesita eliminar las fronteras de cada sector profesional para crear espacios de conocimiento recíprocos y el desarrollo de conocimientos profesionales, en vistas a un mejor seguimiento de la dificultad (Marcel et al., 2007). La coordinación y supervisión de las acciones emprendidas son elementos esenciales para favorecer el desarrollo de un pensamiento y de soluciones complejas para resolver problemas complejos. Como lo indican algunos docentes, la profesión está evolucionando y la colaboración interprofesional ofrece nuevas fuentes de satisfacción profesional.

\section{Limitaciones del estudio y perspectivas}

Colaborar implica orientar la acción de manera colectiva para servir a un objetivo común, explorando, cuestionando incluso las propias repre- 
Medidas socioeducativas y colaboración interprofesional: entre tensiones y nuevas estructuras profesionales

Catherine Blaya, Marco Allenbach, Valérie Angelucci y Frédérique Rebetez

sentaciones mentales, adquiridas durante la socialización profesional de cada uno, en un ambiente de confianza. Además de la voluntad individual, sería importante tener en cuenta los efectos del contexto profesional -en el que se desarrolla la labor de los individuos- sobre las tentativas de colaboración. Algo que no hemos podido hacer en este trabajo, ya que nuestro entorno de investigación se ha limitado a un solo centro.

\section{Referencias}

Allenbach, M (2019). Au cœur de la continuité éducative : le travail dans les espaces inter-métiers, 153-170. In : C. Blaya, C. Tièche-Christinat,\& V.Angelucci (Eds). Au cœur des dispositifs d'accrochage scolaire : continuité et alliances éducatives. BruxeIles, Belgique: EME.

Blaya, C., Gilles, J. L., Plunus, G., \&TiècheChristinat, C. (2011). Accrochage scolaire et alliances éducatives: vers une intégration des approches scolaires et communautaires. Éducation et francophonie, 39(2), 227-249.

Bryk, A. S. (2015). 2014 AERA distinguished lecture: Accelerating how we learn to improve. Educationalresearcher, 44(9), 467-477.

CDHEP, (2002). Code éthique de la recherche pour les Hautes Écoles Pédagogiques. Neuchâtel, Conférence Intercantonale de I'Instruction Publique de la Suisse Romande et du Tessin.Retrievedf rom: https:/www.hepl.ch/files/live/sites/systemsite/files/ centre-soutien-recherche-relations-internationales/pole-levees-fonds/code-ethiquerecherche-rd-2002-hep-vaud.pdf

Crahay, M., Wanlin, P., Issaieva, É., \&Laduron, I. (2010). Fonctions, structuration et évolution des croyances (et connaissances) des enseignants. Revue française de pédagogie. Recherches en éducation, (172), 85-129.

UNESCO (2016). Éducation 2030 : Déclaration d'Incheon et Cadre d'action pour la mise en œuvre de l'Objectif de développement durable 4 : Assurer à tous une éducation équitable, inclusive et de qualité et des possibilités d'apprentissage tout au long de la vie.

Friend, M., Cook, L., Hurley-Chamberlain, D., \&Shamberger, C. (2010). Co-teaching: An illustration of the complexity of collaboration in special education. Journal of Educational and Psychological Consultation, 20(1), 9-27.

Gabola, P., \& Curchod-Ruedi, D. (2017). Une école positive et protectrice pour favoriser la santé des enseignants. Educateur, 2, 16-17.

Henneman, E. A., Lee, J. L., \& Cohen, J. I. (1995). Collaboration: a concept analysis. Journal of Advanced Nursing, 21(1), 103-109.

Kitzinger, J., Markova, I., \& Kalampalikis, N. (2004). Qu'est-ce que les focus groups?. Bulletin de Psychologie, 57(3), 237-243.

Lantheaume, F. (2007). L'activité enseignante entre prescription et réel : ruses, petits bonheurs, souffrance. Éducation et sociétés, 19(1), 67-81.

Lévi-Strauss, Cl. (1990). La Pensée sauvage, Paris, Plon, Pocket, coll. Agora. 
Lynn, C. J., McKay, M. M., \& Atkins, M. S. (2003). School social work: Meeting the mental health needs of students through collaboration with teachers. Children \& Schools, 25(4), 197-209.

Mahieu, P. (1992) Travailler en équipe, Paris, Hachette.

Marcel, J.-F., Dupriez, V., \& Périsset Bagnoud, D. (2007). Le métierd'enseignant : nouvelles pratiques, nouvelles recherches. Dans J.-F. Marcel, V.Dupriez, D. Périsset Bagnoud, \& M. Tardif (Eds).Coordonner, collaborer, coopérer.De nouvelles pratiques enseignantes (p. 7-17). Bruxelles : De Boeck Université.

Maroy, C. (2018). École, régulation et marché: une comparaison de six espaces scolaires locaux en Europe. Presses universitaires de France.

Maroy, C., \&Voisin, A. (2013). Les transformations récentes des politiques d'accountability en éducation : enjeux et incidences des outils d'action publique. Educação\&Sociedade, 34(124), 881-901.

Payet, J. P., Deshayes, F., Rufin, D., et Pelhate, J. (2018). L'enseignant-e, les parents, les spécialistes : diffusion des savoirs experts et altération de la collaboration avec les parents dans I'enseignement prioritaire.Raisons Éducatives (1), 49-73.

Soussi, A., Nidegger, C., Schwob, I., et Genève (canton). Service de la recherche en éducation. (2014). Le rôle des éducateurs des établissements du REP: le point de vue des directeurs d'établissement, des enseignants et des éducateurs. Service de la recherche en éducation.

Tardif, M., et Borgès, C. (2009). Transformations de l'enseignement et travail partagé. Les Sciences de l'éducation-Pour l'Ère nouvelle, 42(2), 83-100.

Thomazet, S. et Mérini, C. (2014) Le travail collectif, outil d'une école inclusive? Questions Vives, 21.

Van Campenhoudt, L. \& Quivy, R. (2011). Manuel de recherche en sciences sociales (4th ed.).Paris:Dunod. 
